\title{
Electroweak Physics Prospects for CDF in Run II
}

Eric James

University of Michigan

for the CDF collaboration

\section{Introduction}

The CDF collaboration will vigorously pursue a comprehensive program of electroweak physics during Run II at the Tevatron based strongly on the successful Run I program. The Run IIa integrated luminosity goal of $2 \mathrm{fb}^{-1}$ will lead to a CDF dataset twenty times larger than that collected in Run I. In addition, an increase in the energy of the colliding beams from $\sqrt{s}=$ $1.80 \mathrm{TeV}$ to $\sqrt{s}=1.96 \mathrm{TeV}$ for Run II provides a $10 \%$ increase in the $W$ and $Z$ boson production cross sections and a corresponding enlargement of the electroweak event samples. In the near term, CDF expects to collect a dataset with 2-3 times the integrated luminosity of Run I by September of 2003.

Utilizing these new datasets CDF will be able to make improved, precision measurements of Standard Model electroweak parameters including $M_{W}, M_{t o p}, \Gamma_{W}$, and $\sin ^{2} \theta_{W}^{e f f}$. The goal of these measurements will be to improve our understanding of the self-consistency of the Standard Model and knowledge of the Higgs boson mass within the model. The top plot in Fig. 1 illustrates our current knowledge of the Standard Model Higgs mass based on measurements of $M_{W}$ and $M_{\text {top }}$. The constraints imposed by combined CDF and D0 Run I measurements of $M_{W}\left(80.456 \pm 0.059 \mathrm{GeV} / \mathrm{c}^{2}\right)$ and $M_{t o p}$ $\left(174.3 \pm 5.1 \mathrm{GeV} / \mathrm{c}^{2}\right)$ are illustrated by the shaded oval region on the plot. The hatched rectangle shows the additional constraint imposed by the recent LEP2 measurement of $M_{W}$. The bottom plot in Fig. 1 illustrates the expected improvement in these constraints based on Run II CDF measurements utilizing a $2 \mathrm{fb}^{-1}$ dataset. The shaded oval region in this plot is based on current estimates of a $40 \mathrm{MeV} / \mathrm{c}^{2}$ uncertainty for measuring $M_{W}$ and a 2-3 GeV/ $c^{2}$ uncertainty for measuring $M_{t o p}$.

In addition to measuring these fundamental parameters, CDF will have the opportunity to take advantage of increased electroweak data samples in Run II to perform precision tests on an assortment of Standard Model predictions. Measurements of $W$ and $Z$ boson production cross sections and the $W$ charge asymmetry are important tests of QCD. The charge asymmetry measurement, in particular, provides useful information on parton distribution functions. In Run II these measurements will be extended into the most 

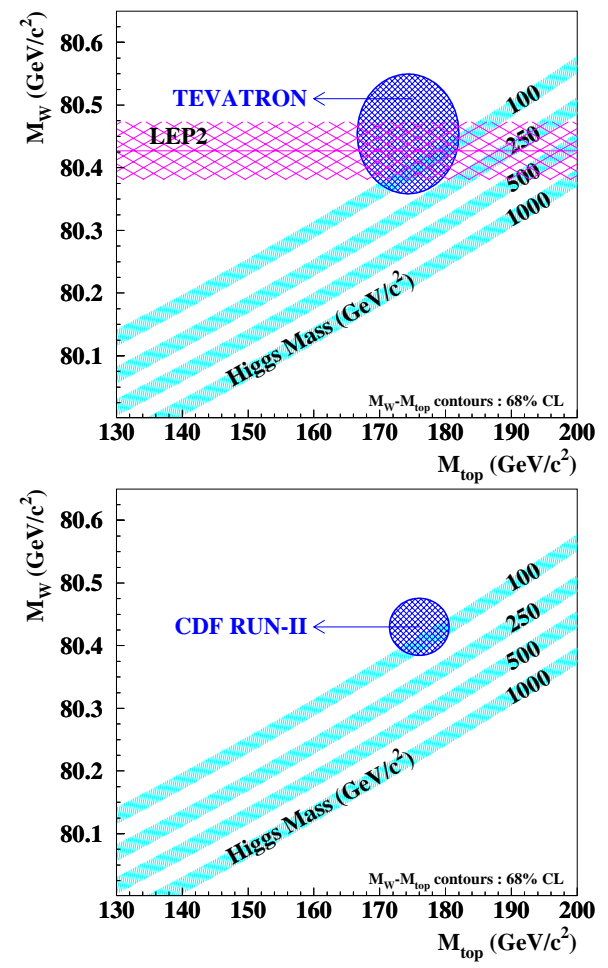

Fig. 1. (Top)Current constraints on the Standard Model Higgs boson mass from measurements of $M_{W}$ and $M_{t o p}$. (Bottom) Expected constraints from Run II CDF measurements based on a $2 \mathrm{fb}^{-1}$ dataset.

interesting range using the increased lepton coverage of the upgraded detector at high $\eta$. With the much larger sample of diboson events to be collected in Run II, CDF will also be able to perform detailed studies of the tri-linear electroweak couplings between the $W, Z$, and $\gamma$ and search for anomalous couplings that could indicate new physics. These types of studies will become more powerful tests of the Standard Model as data samples increase.

\section{$2 W / Z$ Production Cross Sections}

The starting point at the beginning of Run II for the physics program outlined above is re-establishing the pertinent channels in the data and developing a thorough understanding of the upgraded detector. With the small data samples collected prior to June of 2002, CDF has made preliminary measurements of the $W$ boson production cross section using both the electron and muon decay channels. A measurement of the ratio of cross sections $R=\sigma^{W} / \sigma^{Z}$ 
has also been made in the muon channel. The integrated luminosity corresponding to the electron and muon datasets used for these analyses are 10.4 $p b^{-1}$ and $16.5 p b^{-1}$ respectively.

The $W$ bosons used in these analyses are identified in the detector as a reconstructed high $P_{T}$ lepton associated with large missing energy coming from the neutrino in the decay. At the Tevatron the z-component of the neutrino momentum is not observable so all event quantities are measured in the transverse plane. A simple set of kinematic cuts are made to reduce the fraction of background events in the sample. For $W \rightarrow e \nu$ decays the electron is required to be in the central calorimeter $\left(\eta_{e}<1.0\right)$ and have a measured $E_{T}^{e}>25 \mathrm{GeV}$. The missing transverse energy in the event corresponding to $E_{T}^{\nu}$ is also required to be above $25 \mathrm{GeV}$. Similarly, for $W \rightarrow \mu \nu$ decays the muon is required to be in the central muon detectors $\left(\eta_{\mu}<0.6\right)$ and have a measured $P_{T}^{\mu}>20 \mathrm{GeV}$. The corresponding missing transverse energy cut in the muon channel is $E_{T}^{\nu}>20 \mathrm{GeV}$. The small disparities in the kinematic selection criteria between the electron and muon channels are due to different levels of background contamination in the two samples. The leptons in these events are also required to be isolated. The isolation requirement is based on the measured calorimeter $E_{T}$ within a cone of $\Delta R=\left(\Delta \phi^{2}+\Delta \eta^{2}\right)^{.5}=$ 0.4 centered on the reconstructed lepton excluding towers directly along the lepton path. This parameter is required to be less than $4.0 \mathrm{GeV}$ for electrons and less than $2.0 \mathrm{GeV}$ for muons.

The cross section to be measured can be expressed as

$$
\sigma \cdot B=\frac{N_{o b s}-N_{b g}}{A \epsilon \int \mathcal{L} \cdot d t}
$$

where $N_{o b s}$ is the number of observed events, $N_{b g}$ is the estimated number of background events, $A$ is the kinematic and geometrical acceptance, $\epsilon$ is the total efficiency, and $\int \mathcal{L} \cdot d t$ is the integrated luminosity of the data sample.

Background sources for the $W \rightarrow \ell \nu$ event samples include $Z \rightarrow \ell \ell$ events where one lepton is missed by the detector, $W \rightarrow \tau \nu$ events with a subsequent $\tau$ decay into an electron or muon, QCD jet events containing both real and fake muons that falsify a missing $E_{T}$ signature, and cosmic rays (in the muon channel). The total fraction of events originating from background processes in the $W \rightarrow e \nu$ sample is estimated to be $7 \%$ with the largest contribution coming from QCD jet events. In the $W \rightarrow \mu \nu$ sample, the dominant background contribution is from $Z \rightarrow \mu \mu$ events with an unreconstructed muon. The estimate for the total fraction of background events in the muon sample is $12 \%$.

The kinematic and geometrical acceptance $A$ is determined from a Monte Carlo simulation. The representation of the detector geometry in the simulation was verified via direct comparisons with the data. The Monte Carlo was tuned to match the lepton energy resolutions and the distribution of event recoil energies observed in the data. The recoil energy in $W \rightarrow \ell \nu$ 

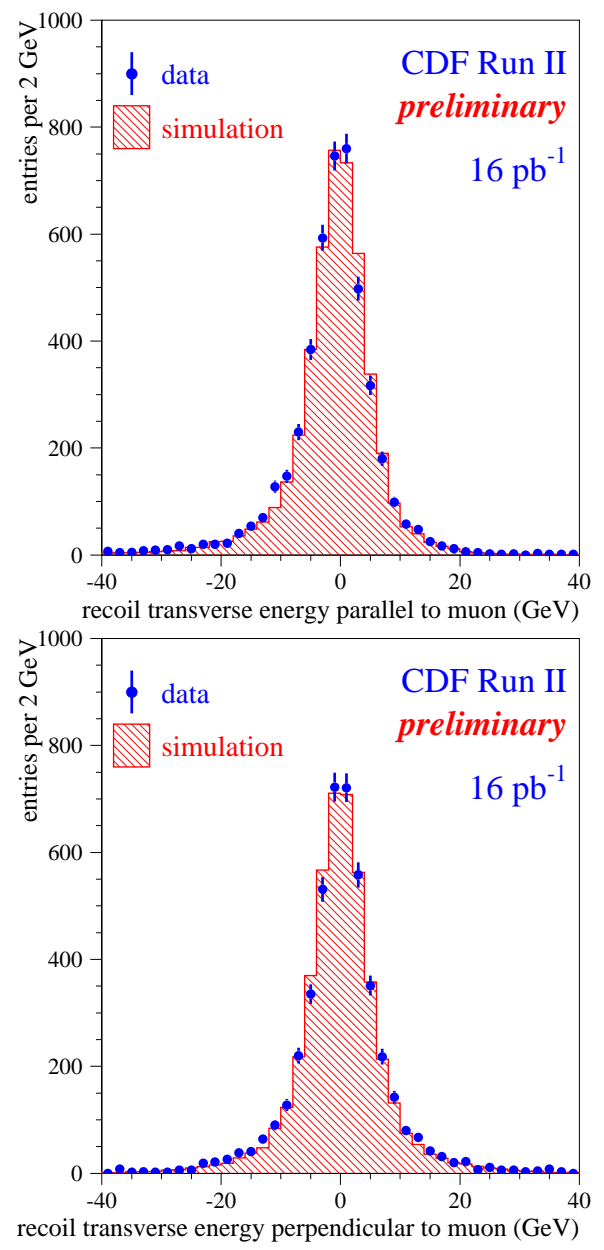

Fig. 2. (Top) Measured recoil energy in $W \rightarrow \mu \nu$ events parallel to the muon direction in Monte Carlo and data. (Bottom)Measured recoil energy in $W \rightarrow \mu \nu$ events perpendicular to the muon direction in Monte Carlo and data.

events is defined as the measured calorimeter energy not assigned to the reconstructed lepton. The recoil energy plays an important role in shaping the missing transverse energy spectrum observed in the data. The importance of the recoil energy model is highlighted by the fact that nearly $20 \%$ of events in these samples have at least one reconstructed jet with $E_{T}>8 \mathrm{GeV}$ and $|\eta|<2.4$. The plots in Fig. 2 show the level of agreement observed between Monte Carlo and data for the recoil energy spectrum in $W \rightarrow \mu \nu$ decays. The top plot shows the measured recoil energy parallel to the direction of the muon and the bottom plot shows the measured recoil energy perpendicular to 
the muon direction. The excellent agreement between data and Monte Carlo seen in these distributions validates the use of our model for extracting the kinematic and geometrical acceptances.

The efficiency $\epsilon$ used in the cross section measurement is a combined number that takes into account several different efficiencies. This number includes efficiencies for triggering on and reconstructing the lepton originating from the $W$ decay; efficiencies for the cuts used to select the good quality beam-produced, isolated leptons from which our samples are constructed; and efficiencies of event topology cuts used to reduce backgrounds in the event samples. The majority of these efficiencies were measured directly from the data. For example, efficiencies of lepton identification and isolation cuts were obtained from studies based on $Z \rightarrow \ell \ell$ events.

Table 1. Cross Section Input Parameters

\begin{tabular}{lll}
\hline & $W \rightarrow e \nu$ & $W \rightarrow \mu \nu$ \\
\hline$N_{\text {obs }}$ & 5547 & 4561 \\
$N_{b g}$ & $409 \pm 85$ & $569 \pm 63$ \\
$A(\%)$ & $23.4 \pm 0.9$ & $14.2 \pm 0.4$ \\
$\epsilon(\%)$ & $81.1 \pm 1.8$ & $63.2 \pm 3.8$ \\
$\int \mathcal{L} \cdot d t$ & $10.4 \pm 1.0$ & $16.5 \pm 1.6$ \\
\hline
\end{tabular}

Table 1 provides a summary of the input parameters to the $W$ production cross section measurements in the electron and muon decay channels. The low efficiency measured for the muon channel is partially due to the use of a non-optimized algorithm for cosmic ray removal that removed roughly $10 \%$ of signal events. This algorithm has already undergone substantial modifications, and the overall efficiency for signal events in the muon channel will be substantially higher in future analyses. The higher acceptance for events in the electron channel is mostly due to differences in the fiducial coverage of the central calorimeter and central muon detectors. The acceptance numbers for both decay channels will increase significantly in future analyses that take full advantage of the improved forward calorimeter and muon coverage in the upgraded CDF detector. The numbers in Table 1 were used to extract preliminary $W$ boson cross section measurements in the electron and muon decay channels. The result based on the electron channel is

$$
\sigma \cdot B(W \rightarrow e \nu)=2.60 \pm 0.03(\text { stat }) \pm 0.13(\text { sys }) \pm 0.26(\text { lum }) n b
$$

and the result based on the muon channel is

$$
\sigma \cdot B(W \rightarrow \mu \nu)=2.70 \pm 0.04(\text { stat }) \pm 0.19(\text { sys }) \pm 0.27(\text { lum }) n b
$$



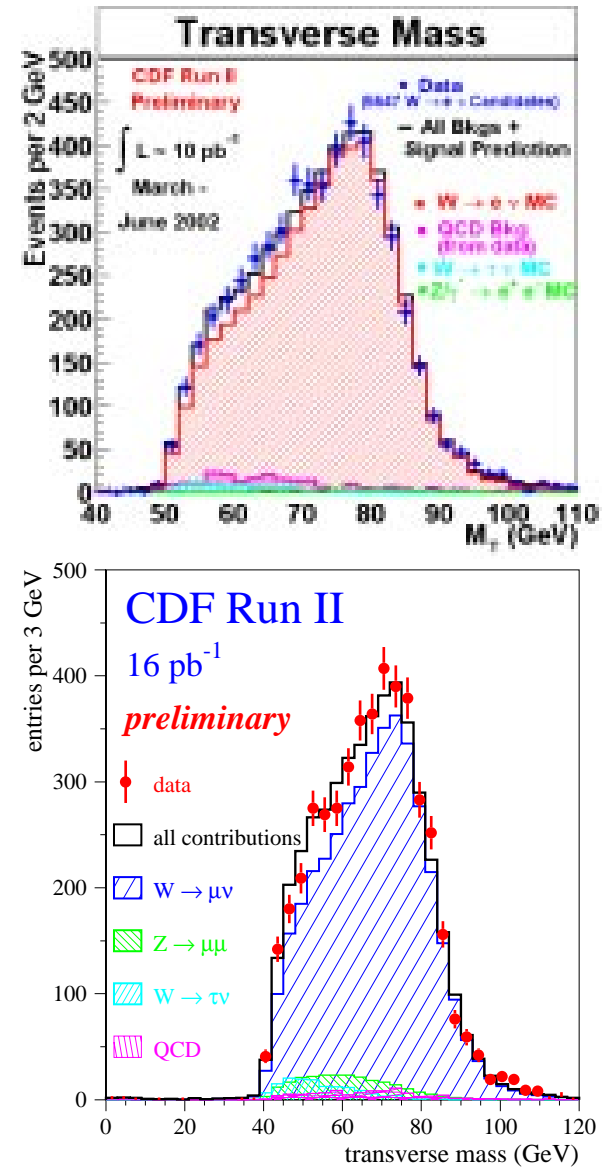

Fig. 3. (Top)Comparison of transverse mass spectrum in data and Monte Carlo for $W \rightarrow e \nu$ events. The shape of the distributions for the signal and background processes are taken from the Monte Carlo and normalized to the expected number of events in the sample. (Bottom)The same distributions for $W \rightarrow \mu \nu$ events.

Both of the these measurements are in good agreement with the recent NNLO theory calculation by Stirling [1] that gives $\sigma \cdot B=2.73 \mathrm{nb}$ at $\sqrt{s}=1.96 \mathrm{TeV}$ and are consistent with an expected $10 \%$ increase in the measured $\mathrm{CDF}$ cross section from Run I [2], $\sigma \cdot B(W \rightarrow e \nu)=2.49 \pm 0.12 n b$ at $\sqrt{s}=$ 1.8 TeV. A final consistency check on the data is made by comparing the transverse mass spectrums in data and Monte Carlo. The top plot in Fig. 3 is for events in the electron channel and the bottom plot is for events in the muon decay channel. The circles with the error bars show the reconstructed transverse mass distributions from the data. The shape of the signal and background distributions are extracted from the Monte Carlo and normalized 
to the expected number of events in the sample. The solid black lines in the plots show the overall distributions obtained from combining the different signal and background distributions. The agreement between the Monte Carlo and data transverse mass spectrums in both samples is excellent. A similar consistency check on the missing $E_{T}$ distributions in data and Monte Carlo was also done but is not shown here due to space considerations.

Table 2. $R_{\mu}$ Input Parameters

\begin{tabular}{ll}
\hline$N_{W}$ & $3992 \pm 93$ \\
$N_{Z}$ & $53.2 \pm 8.0$ \\
$\epsilon_{Z} / \epsilon_{W}$ & $0.884 \pm 0.053$ \\
$A_{Z} / A_{W}$ & $0.2060 \pm 0.0048$ \\
\hline
\end{tabular}

$Z$ bosons used in the measurement of $R_{\mu}$ are identified in the detector as two opposite-sign muons with a combined invariant mass near $90 \mathrm{GeV} / \mathrm{c}^{2}$. For simplicity, both muons are required to pass the same set of kinematic and identification criteria used to select muons for the $W \rightarrow \mu \nu$ event samples. Using this approach, the efficiencies and acceptances extracted for the cross section result can be directly applied to this measurement as well. The ratio of cross sections to be measured can be expressed as

$$
R_{\mu}=\frac{\sigma(p \bar{p} \rightarrow W) \Gamma(W \rightarrow \mu \nu) \Gamma(Z)}{\sigma(p \bar{p} \rightarrow Z) \Gamma(Z \rightarrow \mu \mu) \Gamma(W)}=\frac{N_{W} \epsilon_{Z} A_{Z}}{N_{Z} \epsilon_{W} A_{W}}
$$

Table 2 gives the input values to the measurement of $R_{\mu}$. These values are used to extract a preliminary measurement for the ratio of the $W$ and $Z$ boson cross sections as observed in the muon decay channels. The result is

$$
R_{\mu}=13.66 \pm 1.94(\text { stat }) \pm 1.16(\text { sys })
$$

For comparison, the CDF Run I measurement of this value [3] gave a value of $R=10.90 \pm 0.43$. Due to the small sample of $Z \rightarrow \mu \mu$ events passing our tight selection criteria, this measurement is still statistics-limited in Run II, but this situation will quickly change as more data is collected.

\section{References}

1. W. J. Stirling, private communication.

2. F. Abe et al. (CDF), Phys. Rev. Lett 76, 3070 (1996)

3. F. Abe et al. (CDF), Phys. Rev. D52, 2624 (1995) 